\title{
Zur mathematischen Struktur der lebenden Substanz, dargelegt am Problem der biologischen Temperatur- und Wachstums-Funktion
}

\author{
FRIEDRICH KRÜGER \\ Biologische Anstalt Helgoland, Zentrale, Hamburg-Altona
}

\begin{abstract}
On the mathematical structure of the living substance demonstrated on the basis of the problem of the biological temperature- and growth-function. Some years ago (1961) the author proposed a new formula for the biological temperature function; principally, it is the ARRHENrus formula but the absolute zero is substituted by a lower value, namely, the biological zero temperature. It is not possible to interpret the biological zero temperature on the basis of different velocities of successive reactions. If the ARrmenius function is employed for each link of the chain, the zero temperature $-273^{\circ} \mathrm{C}$ is maintained for the complete chain, resulting in the formulation of a steady state. If we employ a lower value than $-273^{\circ}$ the bending of the curve grows stronger and therefore is better suited for the mathematical description of biological processes. It is shown that the ARRHENrus function may not be applicable if ramifications are present in a chain of biochemical processes. The biological growth function (KRÜGER 1962) is mathematically identical with the temperature function. It is shown that the new formula also is based on the assumption by PÜTTER and BERTALANFFy about the interaction of anabolism and catabolism. It is assumed that the mathematical integration of this statement does not take into consideration the opposite entropy values for anabolism and catabolism. A repeated analysis of more recent values for the growth of man resulted in the discovery of an oscillation in the growth rate superimposed on the basic function. This oscillation causes the well-known acceleration of growth during puberty.
\end{abstract}

\section{EINLEITUNG}

Das Objekt der klassischen Biologie sind Gestalten, deren Form und Mannigfaltigkeit auch heute noch der Mehrzahl der Biologen nur einer Beschreibung, nicht aber einer quantitativen Behandlung zugängig erscheinen. Im Gegensatz zur Morphologie hatte es die Stoffwechselphysiologie von ihrem Beginn an mit Quantitäten zu tun; hieraus wird verständlich, daß wir schon bei RAMEAUX \& SARRus (1837/39) den ersten Versuch einer mathematischen Darstellung ihrer Messungen finden. Die von ihnen aufgedeckte exponentielle Beziehung des Stoffwechsels zum Gewicht war damals sicherlich überraschend. Die vor allem von RubNer (1883) vertretene kausale Deutung durch die Wärmeabgabe an der Körperoberfläche stand bis in die neueste Zeit einer exakten Bearbeitung dieses Phänomens im Wege. Diese Hypothese wurde hinfällig, als sich zeigte (PütTER 1911 und andere), daß die Oberflächenregel in vielen Fällen 
auch für poikilotherme Tiere zutriff. Überdies erwies sich, daß die Oberflächenbeziehung des Stoffwechsels auch bei warmblütigen Tieren nicht streng erfüllt ist (Brody \& Procter 1932).

Um in Zukunft den damals begangenen Fehler zu vermeiden, müssen wir sowohl in der Stoff wechselphysiologie wie auch in der Morphologie immer wieder versuchen, $\mathrm{zu}$ einer möglichst exakten Wiedergabe unserer Meßergebnisse zu kommen. Ich selbst hatte vor einigen Jahren zwei neuartige Formulierungen für die mathematische Beschreibung biologischer Phänomene vorgeschlagen, die eine für die Temperaturabhängigkeit biologischer Prozesse (1961), die andere für die Wiedergabe des tierischen Wachstums (1962). Nachdem gerade letzteres Problem bis in die neueste Zeit für unlösbar gehalten wurde, zeigte sich, daß es mit einem einzigen Ausdruck möglich ist, Wachstumsvorgänge der verschiedensten Art und in weitestem Umfang in sehr guter Näherung wiederzugeben.

Überraschenderweise ergab sich, daß Wachstumsfunktion und biologische Temperaturfunktion mathematisch die gleiche Gestalt haben. Die hieraus hervorgehende Identität des Kurvenverlaufs konnte ich durch den Nachweis erhärten (KRÜGER 1964), daß sich die Wachstumsfunktion von v. BERTALANFFY (1934) auch sehr gut als Temperaturfunktion eignet. Wir begegnen dieser Parallele ebenfalls in der Temperaturfunktion von BELEHRADEK (1930) und der Wachstumsfunktion von MurRay (1925), die mathematisch die gleiche Form haben. Man ist aber nicht berechtigt, hieraus irgendwelche Schlïse zu ziehen, da es sich bei der Temperaturfunktion um die Wiedergabe von Geschwindigkeiten handelt, bei der Wachstumsfunktion dagegen um die Darstellung von Dimensionen. Die Wachstumsgeschwindigkeit wird in letzterem Falle durch den Differentialquotienten wiedergegeben, der eine andere Form hat. Die formale Ubereinstimmung beruht darauf, daß es sich in beiden Fällen um Vorgänge handelt, die sich bei logarithmischer Darstellung asymptotisch einem Maximalwert nähern.

\section{DEUTUNG DES KURVENVERLAUFS BIOLOGISCHER TEMPERATURFUNKTIONEN}

Die von mir vorgeschlagene Temperaturfunktion:

$$
y_{t}=\frac{m}{n^{\frac{1}{t-z}}}
$$

steht in engster Beziehung zu der im anorganischen Bereich gültigen ArrhenIUsFunktion. Sie unterscheidet sich von ihr dadurch, daß an Stelle der absoluten Nullpunkt-Temperatur von $-273^{\circ} \mathrm{C}$ ein geringerer Wert als Bezugstemperatur eingesetzt wird. Diese biologische Nullpunkt-Temperatur im Nenner des Exponenten bezeichne ich mit dem Symbol z. Der rechnerisch eingesetzte $z$-Wert ist mathematisch ein Ausdruck für die Krümmung der Kurve, die man erhält, wenn man die Logarithmen der Meßwerte den linearen Temperaturwerten auf der Abszisse gegenüberstellt. Gegenüber früheren Vorschlägen zur mathematischen Darstellung biologischer Temperaturbeziehungen (JøRgensen 1916, JANISCH 1927, BELEHRADEK 1930), deren rein deskriptive Eignung nicht bestritten werden soll und kann, besitzt die neue Formel einige Vor- 
züge und schließt vor allem a priori die Arrhentus-Funktion ein, die mit Sicherheit in manchen Fällen auf biologische Prozesse anzuwenden ist. Außerdem gibt die neue Funktion - soweit sich bislang erkennen läßt - den Kurvenverlauf biologischer Temperaturfunktionen etwas besser wieder (Krüger 1964). Schließlich scheint die neue Funktion den Vorzug zu bieten, daß man von ihr ausgehend auch einen mathematisch allerdings noch nicht vollkommen durchschaubaren - Anschluß an die Wachstumsfunktion erreichen kann (KRÜGER 1963).

Die Bedeutung der in der Formel enthaltenen Nullpunkt-Temperatur z versteht man am einfachsten an Hand einer graphischen Darstellung (Abb. 1). Stellt man die

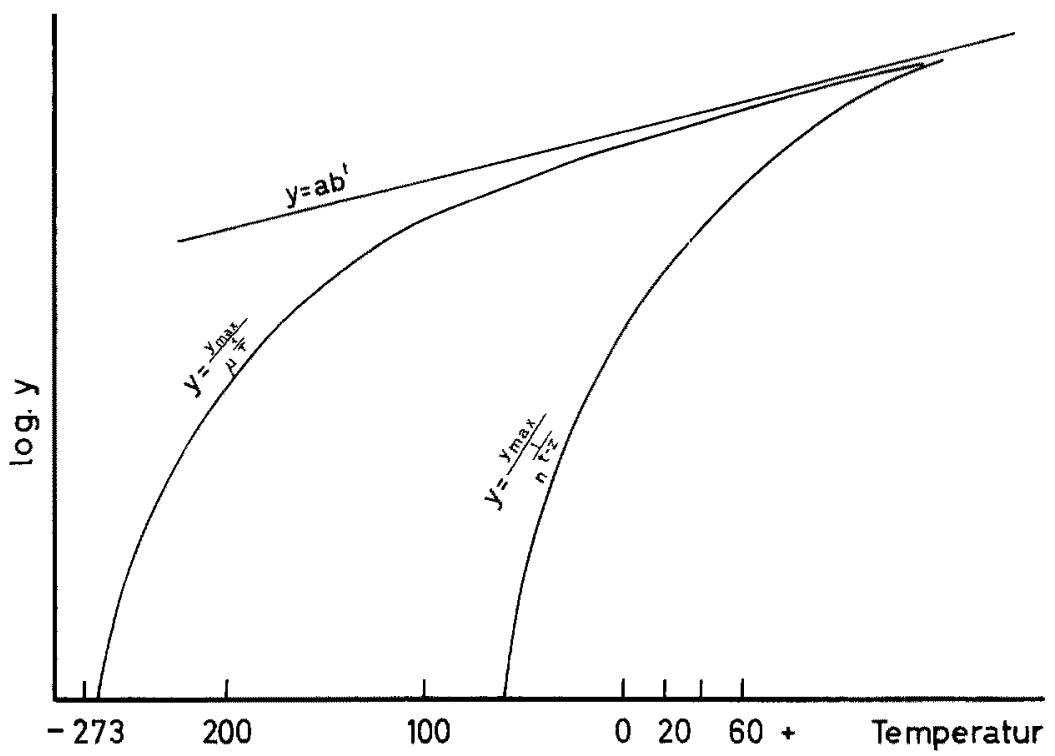

Abb. 1: Schematischer Vergleich des Kurvenverlaufs entsprechend der RGT-Regel; ARRHENIUSFunktion und eigener Vorschlag

Logarithmen der Meßwerte auf der Ordinate als Funktion der linearen Meßwerte auf der Abszisse dar, so erhält man eine Gerade, wenn die Werte der BERTHELot-Funktion folgen, die der RGT-Regel zugrunde liegt. Für die ARRHenrus-Funktion ergibt sich eine Kurve, die im biologischen Temperaturbereich nur schwach gekrïmmt ist und daher der Berthelot-Geraden sehr nahe kommt. Erst unterhalb des biologisch interessierenden Temperaturbereichs ist die ARRHENIUs-Kurve stärker gekrümmt und fällt schließlich zum absoluten Nullpunkt überaus steil ab. Setzen wir aber in der ARRHENIUSFunktion eine höhere Temperatur als den absoluten Nullpunkt ein, so verschiebt sich entsprechend der stärker gekrümmte Abschnitt der ARRHENIUs-Kurve in den biologischen Temperaturbereich und gestattet auf diese Weise eine Anpassung an gegebene Meßdaten. In solchen Fällen durch verschiedene $\mathrm{Q}_{10^{-}}$oder $\mu$-Werte den Gang der Temperaturkurve wiederzugeben, ist mathematisch vollkommen sinnlos und kann daher auch nicht zu auswertbaren Zahlen führen. 
Die der neuen Temperaturfunktion folgende Kurve endet bei $-z^{0}$ auf der Abszisse. Durch Veränderung dieses Wertes kann man den Kurvenverlauf weitgehend verändern und an gegebene Meßreihen anpassen. Gleichzeitig charakterisiert man durch $z$ die Krümmung der Kurve. Es ergibt sich nun das Problem, wie wir den $z$-Wert - die biologische Nullpunkt-Temperatur - deuten können. Zunächst vermutete ich - im Anschluß an Crozier (1924/25) -, daß durch die Hintereinanderschaltung von Reaktionsstufen mit verschiedener Geschwindigkeit die Krümmung der biologischen Temperaturkurven zu erklären sei. Nehmen wir aber die Arrhentus-Funktion als mathematische Basis an, so ergibt sich bei der mathematischen Darstellung einer einfachen Kette hintereinandergeschalteter Reaktionen mit verschiedener Geschwindigkeitskonstante $\mu$ die Gleichung eines Fließgleichgewichtes, bei dem die Gesamtgeschwindigkeit gleich dem Produkt der einzelnen Geschwindigkeitskonstanten ist. Der absolute Nullpunkt bleibt aber als Bezugstemperatur erhalten. Auf diesem Wege, das heißt also, durch die Aufeinanderfolge von Reaktionen mit verschiedener Geschwindigkeit, die nacheinander als Schrittmacher-Reaktionen auftreten, können wir die bei biologischen Messungen gefundenen Anderungen der $\mu$-Werte nicht erklären. Hiermit wird also auch die Hypothese von Crozier hinfällig und damit der Aussagewert der berechneten $\mu$-Werte.

Ich habe mir nun die Frage gestellt, wie die Verhältnisse liegen, wenn wir nicht von einer einfachen Kette hintereinandergeschalteter Reaktionen ausgehen, sondern eine Verzweigung in der Kette auftritt, wenn wir also annehmen, daß von einem gegebenen Substratbestand zwei Reaktionen ausgehen. Diese Bedingung muß man im Stoffwechselgeschehen des Organismus sicherlich als gegeben ansehen. Wir gehen hierbei von der Vorstellung aus, daß für jede der Reaktionen die Arrhenrus-Funktion zutriff, und legen die von ARrhenius gegebene Interpretation der Parameter zugrunde. Im Zähler der Formel steht danach die Gesamtmenge der zur Verfügung stehenden Substanz - m -, im Nenner die Geschwindigkeitskonstante - $\mu$ - hoch dem reziproken Wert der absoluten Temperatur. Der Nenner repräsentiert den Anteil von m, der bei der gegebenen Temperatur nicht an der Reaktion beteiligt ist. Der Quotient beider Größen stellt y : den reaktionsfähigen Anteil von $\mathrm{m}$ dar. Nehmen wir nun den Fall einer Verzweigung an, so steht für die einzelne Reaktion nicht mehr die gesamte Menge des Vorrates m zur Verfügung, sondern nur der um den Anspruch der zweiten Reaktion verminderte Anteil. Dementsprechend wäre für die beiden Reaktionen anzusetzen:

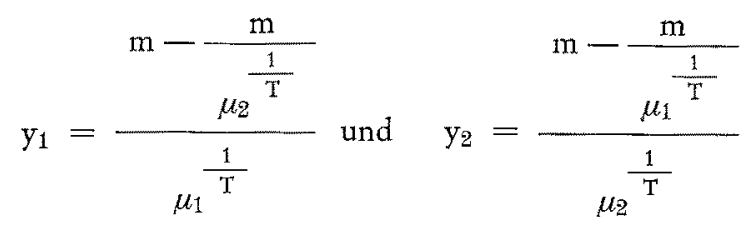

Durch das Auftreten des Temperaturwertes im Zähler verliert bei Verzweigungen in der Reaktionskette die ARRHENIUS-Funktion offensichtlich ihre Anwendbarkeit, die eine einfache Proportionalität zum reziproken Wert der absoluten Temperatur fordert. Die Bezugstemperatur von $-273^{\circ} \mathrm{C}$ ist in diesem Fall nicht mehr zutreffend. Die $\mathrm{Ab}$ weichung von der ARrHenIus-Funktion ist offensichtlich dadurch bedingt, daß die zwei 
nebeneinander von dem Substratvorrat zehrenden Reaktionen den reaktionsfähigen Anteil schon bei höheren Temperaturen und nicht erst beim absoluten Nullpunkt erschöpfen lassen. Es gibt aber auch noch andere Denkmöglichkeiten, die den für den Reaktionsablauf mitbestimmenden Substratvorrat schon bei höheren Temperaturen reduzieren könnten, wie zum Beispiel Diffusionshemmungen. Diese Oberlegungen sollen nur zeigen, daß wir zwanglos die Tatsache deuten können, daß für biologische Reaktionsabläufe im allgemeinen die ArrHenIUs-Funktion nicht in ihrer ursprünglichen Form anwendbar ist.

Für den Organismus ergibt sich hieraus die Möglichkeit, durch die Einschaltung von Verzweigungen in den Reaktionsketten die Form der Temperaturabhängigkeit seiner Stoffwechselprozesse zu ändern und abweichend vom Geschehen im anorganischen Bereich zu gestalten. Diese Möglichkeit ist für den Organismus dadurch bedeutungsvoll, daß er in die Lage versetzt wird, seinen Stoffwechsel an die Temperaturbedingungen seines Biotops anzupassen.

\section{DIE WIEDERGABE VON ANABOLISMUS UND KATABOLISMUS ALS FUNKTION DER ZEIT}

An einem Beispiel (Salvelinus fontinalis) hatte ich früher gezeigt (1961, 1963), daß die Berechnung der Parameter der neuen Temperaturfunktion für Individuen unterschiedlicher Größe zu Zahlen führt, die eine verhältnismäßig einfache mathematische Beziehung zum Gewicht erkennen lassen. Die hiermit aufgedeckte Beziehung der Parameter zum Gewicht stellt aber primär eine Altersabhängigkeit dar. Dieser Umstand war für mich der Anlaß, das Problem der mathematischen Darstellung von Wachstumswerten aufzugreifen. Die graphische Analyse von Wachstumsdaten führte (1961, 1965) zu dem der Temperaturfunktion entsprechenden Ausdruck:

$$
\mathrm{d} \chi=\frac{\mathrm{D}_{\mathrm{max}}}{\mathrm{N}^{\frac{1}{\chi+\xi}}}
$$

$(\mathrm{d} \chi=$ Dimension im Alter $\chi) ; \mathrm{D}_{\max }=$ Maximalgröße; $\mathrm{N}=$ Geschwindigkeitskonstante; $\xi=$ additiver Zeitwert) ${ }^{1}$.

Die neue Funktion besitzt vor der Mehrzahl der älteren Vorschläge den entscheidenden Vorzug, daß sie bei sehr guter Näherung an gegebene Wachstumswerte in einer einfachen mathematischen Beziehung zur allometrischen Funktion steht. Diese stellt eine durch ungezählte Beispiele gesicherte mathematische Beschreibung des relativen Wachstums dar. Der besonders bedeutungsvolle Exponent $a$ dieser Gleichung stellt den Quotienten der. Logarithmen der Geschwindigkeitskonstanten der beiden miteinander verglichenen Wachstumsvorgänge dar:

$$
a=\frac{\log N_{1}}{\log N_{2}}
$$

Durch diesen Zusammenhang haben wir jetzt die Möglichkeit, mit den Parametern der

${ }^{1}$ Erst nach Fertigstellung des Manuskriptes fand ich, daß schon früher von ZucKer (1941) ein resiproker Alterswert zur Darstellung des Gewichts-Wachstums der Ratte benutzt wurde. 
Mathematische Struktur der lebenden Substanz

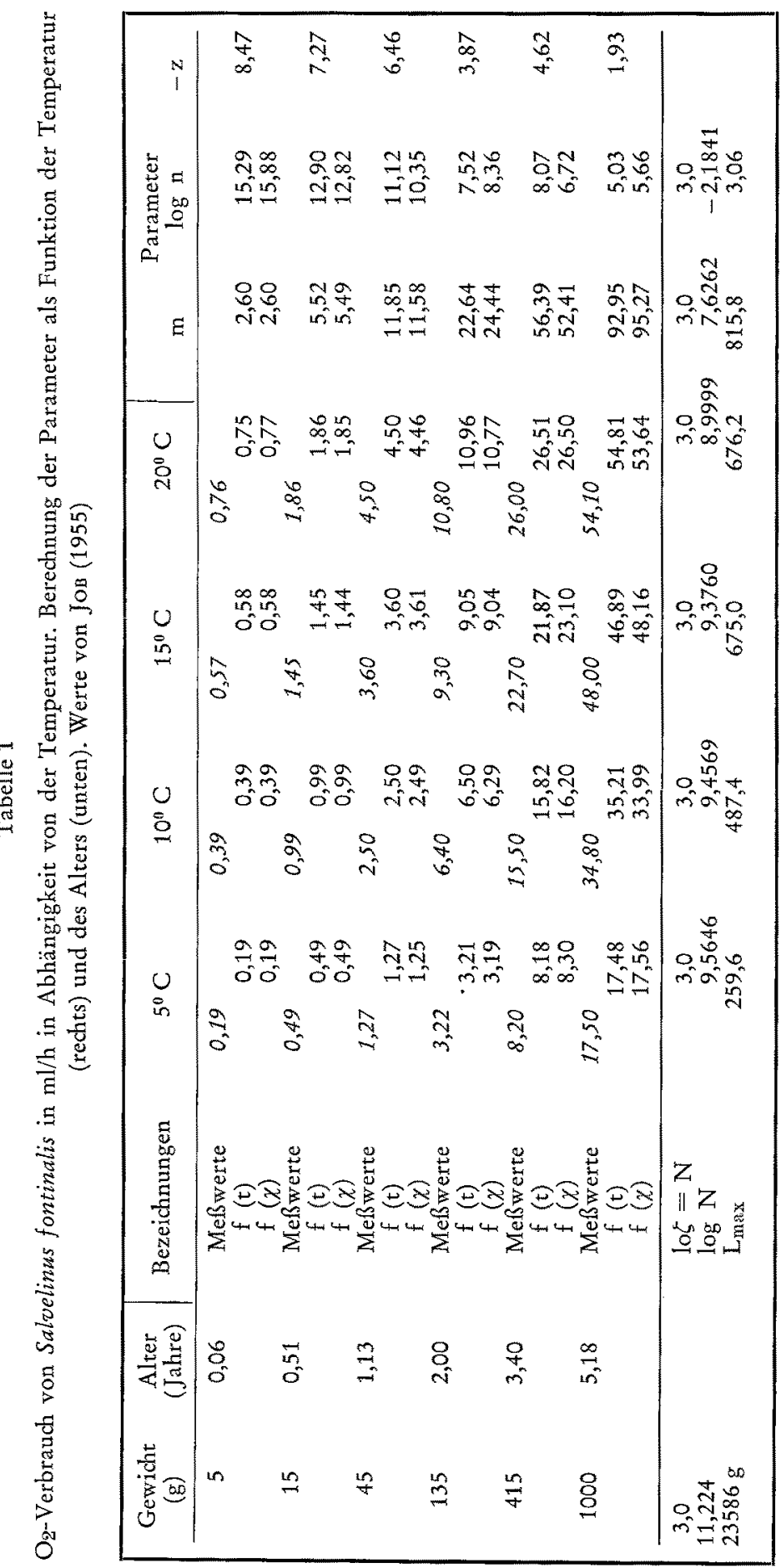


allometrischen Funktion in die Wachstumsfunktion überzugehen und umgekehrt und haben damit ein gewisses erstes Gerüstsystem in der mathematischen Behandlung biologischer Erscheinungen gewonnen. Da sich die allometrische Funktion nicht nur auf morphologische Daten bezieht, sondern ebensogut auch auf Stoffwechselwerte angewandt werden kann, ist ebenfalls eine Brücke zwischen diesen Gebieten geschaffen.

Auf dem ersten Symposium hatte ich gezeigt, daß wir bei Anwendung der neuen Funktion auf Wachstumsvorgänge fast zu den gleichen Zahlen kommen, wie sio sich aus der Bertalanffy-Funktion errechnen. Die Differenzen liegen weit unterhalb der Meßfehler. Hierdurch tauchte die Frage nach der Beziehung der Bertalanffy-Funktion zu meinem eigenen Vorschlag auf.

Von BertalanfFy ging bei der Aufstellung seiner Funktion (1934) - ebenso wie schon vor ihm PütTer (1920) - von der Annahme aus, daß das Wachstum aus zwei entgegengesetzten Stoffwechselprozessen resultiert: dem Aufbaustoffwechsel oder Anabolismus und dem Abbaustoffwechsel oder Katabolismus. Für ersteren nehmen beide Autoren an, daß er - wenigstens näherungsweise - der Oberflächenentwicklung, also der $2 / 3$-Potenz folgt, während die Abbauprozesse gewichtsproportional verlaufen. Für beide Prozesse wird die Gültigkeit der allometrischen Bezichung zugrunde gelegt. Das bedeutet, daß bei Abtragung der Logarithmen von Anabolismus und Katabolismus als Funktion des Logarithmus des Gewichtes in einem rechtwinkligen Koordinatensystem, beide Vorgänge auf Geraden liegen, deren Steigungswinkel für den Anabolismus etwa $34^{\circ}$ und für den Katabolismus $45^{\circ}$ beträgt. Beide Geraden schneiden sich dort, wo Anabolismus und Katabolismus den gleichen Wert haben. Dieses ist der Maximalwert der Ber'talanfFy-Funktion. Hinter diesem Punkt, der vom Organismus aber nie erreicht wird, würde ein negatives Wachstum resultieren.

Benutzen wir nun an Stelle der logarithmischen Unterteilung auf der Abszisse, welche der allometrischen Darstellung zugrunde liegt, eine Unterteilung in die reziproken Alterswerte, wie sie der von mir vorgeschlagenen Funktion entspricht, so ändert sich hierdurch nichts an der Beziehung zwischen den Wertepaaren von Anabolismus und Katabolismus für die einzelnen Gewichtsstufen. Diese werden aber nun zu Altersstufen, und durch die Verschiebung auf der Abszissenteilung werden aus der Geraden Kurven, die für den Alterswert $\infty$ dem gleichen Maximalwert zustreben. Der Maximalwert ist also für beide Teilprozesse identisch. Auch für $\mathrm{N}$ können wir den gleichen Wert einsetzen, wenn wir entsprechend Gleichung (3) die Exponenten des Nenners mit den entsprechenden $\alpha$-Werten multiplizieren. Auf der Basis der neuen Funktion würde dann der Pütrer-Bertalanffy-Ansatz die Form annehmen:

$$
\frac{d w}{d t}=\frac{\operatorname{Max}}{N^{\frac{2}{\tau}}}-\frac{\operatorname{Max}}{N^{\frac{3}{\tau}}}
$$

(Bei der Identität der Temperatur- und Wachstumsfunktion benutze ich für Zeitwerte griechische Symbole, um Verwechslungen zu vermeiden. $\tau$ bedeutet in diesem Falle die Summe von Geburtsalter $\chi$ plus dem additiven Zeitwert $\xi$.) Die Funktionen für Anabolismus und Katabolismus unterscheiden sich also nur durch den Zähler des Exponenten; alle anderen Parameter sind gleich. 
Grundsätzlich muß man wohl den PütTer-Bertalanffy-Ansatz als richtig ansehen. Es sprechen hierfür nicht nur die zugrunde gelegten experimentellen Befunde, sondern vor allem die Tatsache, daß er in der bekannten Wachstumsformulierung zu einer ausgezeichneten Wiedergabe von Wachstumsdaten als Funktion der Zeit geführt hat. Wenn die mathematische Integration des Ansatzes nicht zu einer vollkommen befriedigenden Lösung führte, muß man annehmen, daß er nicht in vollem Umfang die vorliegenden Beziehungen wiedergibt. Vermutlich liegt ein Fehler darin, daß er die Vorgänge von Auf- und Abbau der Körpersubstanz proportional den zugrunde liegenden Stoffwechselprozessen annimmt. Hier ist sicherlich eine Korrektur anzubringen. Der Anabolismus läuft unter Entropieverminderung $a b$, und die für die Entropiebindung aufgewandte Energie kann beim Katabolismus nicht wieder nutzbar gemacht werden. Der Einfluß dieses Faktors bleibt zu prüfen. Der BERTALANFFY-Ansatz gilt nur für die Massenänderungen, nicht aber in dieser Form für die ihnen zugrunde liegenden Stoff wechselprozesse*.

\section{DARSTELLUNG DER TEMPERATURABHANGIGKEIT DES STOFFWECHSELS ALS FUNKTION DES ALTERS}

Die Wiedergabe des Bertalanffy-Ansatzes als Funktion des Alters führte, wie wir sahen, zu einer wesentlichen Vereinfachung gegenüber dem Bezug auf das Gewicht. Das ist nicht erstaunlich, da ja das Gewicht selbst eine Funktion des Alters darstellt, die beim direkten Bezug auf das Alter in Fortfall kommt. Aus diesem Grund unternahm ich den Versuch, die in früheren Untersuchungen ausgewerteten Zahlen von JoB (1955) für die Temperaturabhängigkeit der Atmung des Fisches Salvelinus fontinalis als Funktion des Alters darzustellen. Man erhält dann die Parameter der Temperaturabhängigkeit des Stoffwechsels für die verschiedenen Altersstufen.

Јов hat die Atmungsgröße als Funktion des Gewichtes seiner Fische wiedergegeben. Aus Wachstumsdaten in der Literatur habe ich versucht, zu den gegebenen Größenklassen das Alter zu bestimmen (CARLANDER \& Ricker 1962, Bridges \& Mullan 1958). Die verschiedenen Wachstumsdaten für Salvelinus stimmen allerdings nicht ganz überein. Eine gute Näherung an die meisten Daten erhält man mit einem Wert von 3 für den additiven Alterswert $\xi$. Da $\xi$ für die Berechnung nicht sehr kritisch ist, ändern geringere Abweichungen grundsätzlich nichts an den Ergebnissen. Mit Kenntnis der Wachstumsparameter läßt sich leicht zum angegebenen Gewicht das Alter der Fische berechnen. Es kommt gut mit den Literaturwerten überein. Für alle Temperaturen wurde der gleiche $\xi$-Wert 3,0 eingesetzt, da man ja die Vorgeschichte der Versuchstiere als identisch annehmen muß. Die Berechnung ergab für $\log \mathrm{N}$ bei allen vier Temperaturen Werte gleicher Größenordnung, die bemerkenswerterweise mit steigender Temperatur fallen (Tab. 1). Ihre Temperaturabhängigkeit könnte man näherungsweise schon durch eine lineare Funktion wiedergeben. Die geringe Temperaturabhängigkeit der Werte für $\log N$ stimmt mit der relativen Konstanz der von JoB berechneten Exponenten der allometrischen Funktion überein. Starke Differenzen zeigen nur die Maximalwerte, die mit steigender Temperatur höher werden.

\footnotetext{
* Es soll das Problem in Kürze in einer besonderen Untersuchung behandelt werden.
} 
Auch dieses Beispiel zeigt die wesentliche Vereinfachung der mathematischen Beziehungen, wenn wir die Meßwerte nicht auf das Gewicht, sondern auf das Alter beziehen. Vor allem aber sollen hiermit die interessanten und aufschlußreichen Möglichkeiten einer mathematischen Auswertung von Stoffwechselversuchen auf der Basis der neuen Wachstumsfunktion aufgezeigt werden. Soweit ich erkennen kann, sind sie mit anderen Wachstumsformulierungen nicht gegeben.

\section{DIE MATHEMATISCHE WIEDERGABE DES WACHSTUMS DER RATTE}

Abschließend möchte ich noch auf einige Anwendungen der neuen Funktion auf Wachstumsdaten aus der Literatur zu sprechen kommen, um ihren weiten Geltungs.bereich zu demonstrieren. Offensichtlich ist ihre Einsatzmöglichkeit nicht auf das Wachstum vielzelliger Organismen begrenzt, sondern umfaßt auch das Zellwachstum. Tabelle 2 zeigt die Auswertung der Zahlen von BordzILOWSKaJA, einer Schülerin von Schmalhausen, über das Wachstum von Hefezellen. Es ergab sich eine überaus gute Ubereinstimmung von beobachteten und berechneten Zahlen. Dieses Beispiel hatte übrigens auch schon v. BERTALANFFY (1934) auf der Basis seiner Formulierung erfolg-

Tabelle 2

Wachstum der Hefe (Werte von BordzilowsKaja aus SchMalmausen 1929)

\begin{tabular}{|c|c|c|}
\hline Zeit & Länge & $\begin{array}{c}\begin{array}{c}\text { Länge } \\
\text { (berechnet) }\end{array} \\
\zeta=0,408 \\
\log \mathrm{N}=0,3295 \\
\mathrm{~L}_{\max }=88,14\end{array}$ \\
\hline $\begin{array}{r}0 \\
10 \mathrm{~min} \\
40 \mathrm{~min} \\
1 \mathrm{~h} \\
20 \mathrm{~min} \\
40 \mathrm{~min} \\
2 \mathrm{~h} \\
20 \mathrm{~min} \\
40 \mathrm{~min} \\
3 \mathrm{~h}\end{array}$ & $\begin{array}{l}13,70 \\
31,06 \\
42,83 \\
51,53 \\
57,15 \\
61,14 \\
64,24 \\
66,63 \\
68,23 \\
70,57\end{array}$ & $\begin{array}{l}13,72 \\
31,62 \\
43,50 \\
51,42 \\
57,00 \\
61,14 \\
64,32 \\
66,83 \\
68,86 \\
70,54\end{array}$ \\
\hline
\end{tabular}

reich darstellen können. Ebenso lassen sich auch die Zahlen von ScHMaLHAusen (1925) über das Wachstum von Paramecium zwischen zwei Teilungsschritten mit der neuen Funktion sehr gut wiedergeben.

Die neue Wachstumsfunktion leitete ich an Daten für das Wachstum von Fischen ab. Bei ihnen dürfen wir ein unbegrenztes Wachstum annehmen, das also während des ganzen Lebens andauert. Bei den Säugetieren, deren Wachstum nicht nur von theoretischem, sondern auch von praktischem Interesse ist, haben wir es dagegen mit einem begrenzten Wadstum zu tun, das also beim Erreichen einer gewissen Endgröße aufhört. Hier ergibt sich die Frage, in welcher Form das Wachstum abgebremst wird. 
Als Musterbeispiel wählte ich das Wachstum der weißen Ratte, das Objekt zahlreicher Untersuchungen war und daher als relativ gut bekannt vorausgesetzt werden darf. Es stellt dieses eine wesentliche Voraussetzung für die mathematische Verarbeitung von Wachstumsdaten dar. Als spezielles Beispiel wählte ich die Zahlen von Zucker \& Zucker (1962) für das Gewichtswachstum von Sherman-Ratten. Die Auswertung der Daten für das gesamte Wachstum führte zu keinem brauchbaren Ergebnis. Daher schied ich die Werte für die älteren Tiere aus. Bei der starken Krümmung der Wachstumskurve im unteren Bereich gestattet auch eine geringere Zahl von Werten eine zuverlässige Bestimmung von $\xi$ als dem entscheidenden Parameter für die Berechnung.

Tabelle 3

Gewichtswachstum von SHERMAN-Ratten (Werte von ZUCKER \& ZUCKER 1962)

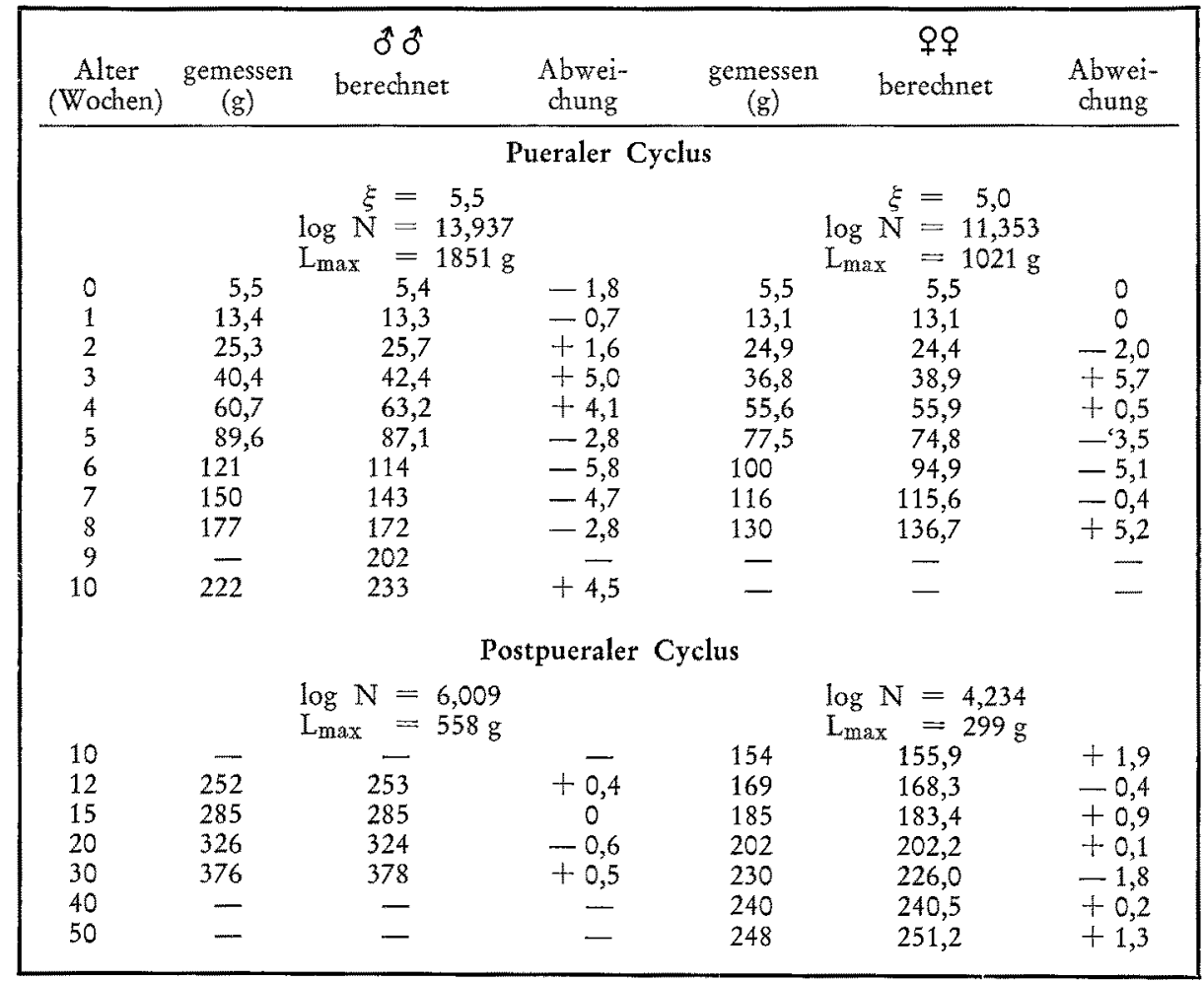

Mit dem gefundenen Wert von 5,5 Wochen für die $\delta \hat{\delta}$ und 5 Wochen für die $q \phi$ ließs sich das Wachstum bis zur Geschlechtsreife - das heißt zehn Wochen bei den $\hat{\delta} \hat{\partial}$ und acht Wochen bei den 우 - sehr gut wiedergeben, wie Tabelle 3 zeigt. Die grafische Darstellung dex ganzen Gewichtsdaten auf der Basis der errechneten $\xi$-Werte (Abb. 2) zeigte nun, daß sich hierbei auch die der Pubertät folgenden Gewichte auf einer Geraden anordnen, deren Steigung aber wesentlich geringer ist. Hieraus ergab sich die wichtige Erkenntnis, daß wir für das gesamte postnatale Wachstum der Ratte bei der Berechnung einen einheitlichen $\xi$-Wert einsetzen können, nur daß nach der Ge- 
schlechtsreife das Wachstum mit stark verminderter Geschwindigkeit abläutt. Im Zusammenhang mit der Reduktion der Wachstumsgeschwindigkeit - mathematisch aus-
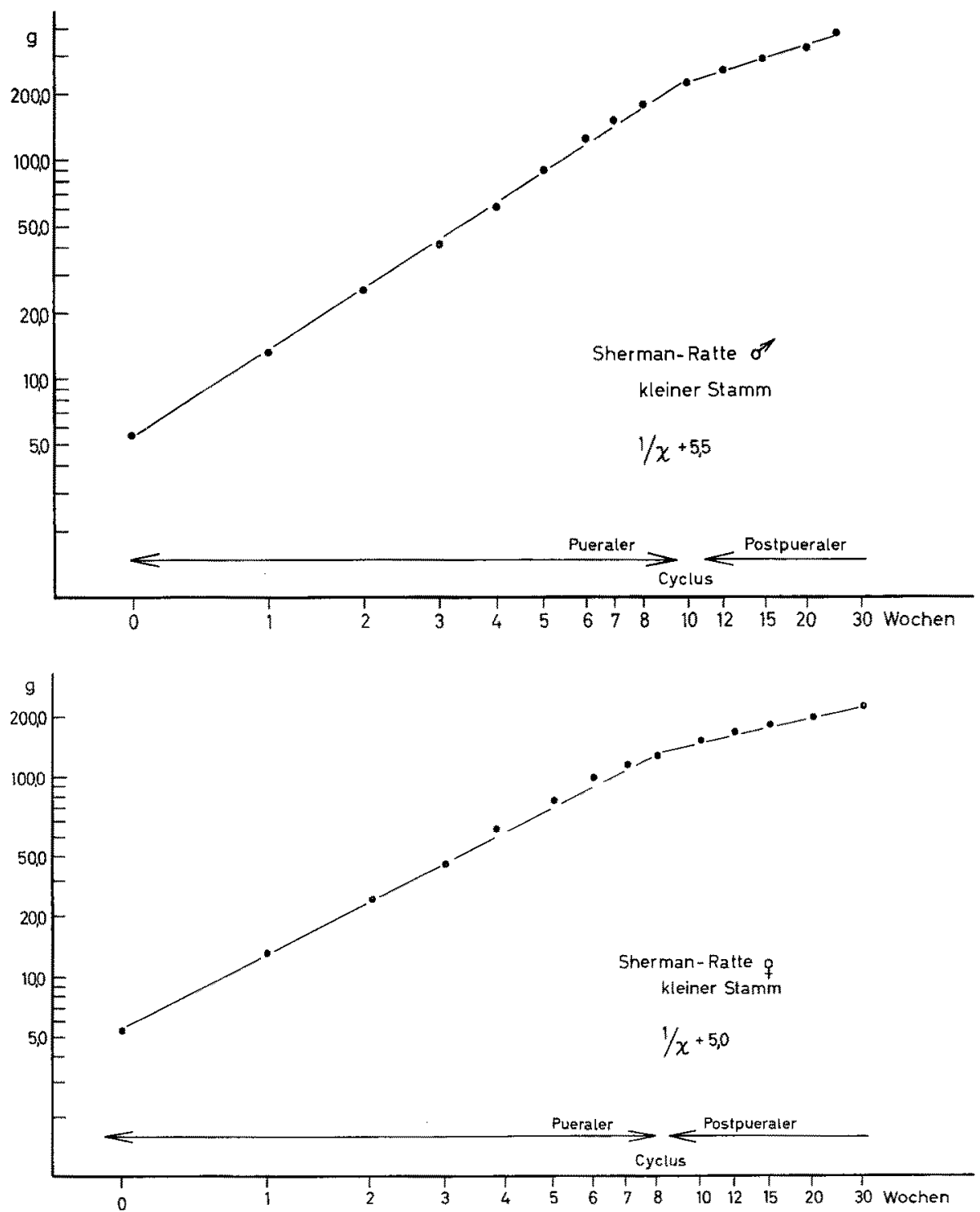

Abb. 2: Darstellung des Gewichtswachstums von Sherman-Ratten (kleiner Stamm) gemäß der neuen Funktion $(\xi=5,5-$ beziehungsweise 5,0 Wochen). Die Verlangsamung des Wachstums nach 10 beziehungsweise 8 Wochen tritt deutlich hervor (Werte von Zucker 1962)

gedrückt durch die Geschwindigkeitskonstante N - wird auch der Wert für die Maximalgröße reduziert. Mit fortschreitender Annäherung an den Maximalwert verringern sich auch die Zuwachsraten, so daß sie schließlich praktisch unmeßbar klein 
werden: Das Wachstum klingt aus. Die Zahlen von Zucker et al. (1941) lassen erkennen, daß mit einem Alter von etwa 70 Wochen das Gewichtswachstum noch nicht vollkommen zum Stillstand gekommen ist, wenn auch die Einzelwerte stark schwanken.

Die an diesem Beispiel aufgezeigte Notwendigkeit, das postnatale Wachstum in zwei Cyclen zu unterteilen, wurde auch schon von v. BERTALANFFY und anderen Autoren erkannt. BertalanfFy benutzt hierfür die Bezeichnung (1960) „praepubertaler" und "postpubertaler" Cyclus. Ich ziehe vor, von einem "pueralen" und "postpueralen" Cyclus zu sprechen, wobei ich die beiden Cyclen - im Hinblick auf die Verhältnisse beim Menschen - als „puerale Wachstumsperiode" zusammenfasse. Sie steht im Gegensatz zur "embryonalen Wachstumsperiode“, für die auch bei der Ratte andere Parameter - vor allem ein geringerer $\xi$-Wert - einzusetzen sind.

Der an diesem Beispiel gefundene $\xi$ Wert läßt sich auch für die Wiedergabe anderer Wachstumsreihen der Ratte einsetzen. Es handelt sich hierbei nicht um einen mathematisch exakt berechneten, sondern auf 0,5 abgerundeten Näherungswert.

\section{ZUR MATHEMATIK DES MENSCHLICHEN WACHSTUMS}

Die bei der Auswertung des Rattenwachstums gewonnene Erkenntnis, daß man bei den Säugetieren mit dem Vorkommen von zwei Wachstumscyclen rechnen muß, die sich auf der Basis eines konstanten $\xi$-Wertes hinsichtlich der Geschwindigkeitskonstante und im Zusammenhang damit auch des Maximalwertes unterscheiden, muß man auch bei der Bearbeitung menschlicher Wachstumsdaten berücksichtigen. Hierauf hatte ich bei meinem ersten Versuch, über den ich früher berichtete (KRÜGER 1964), noch nicht achten können. Durch die Einbeziehung von Werten des postpueralen Cyclus hatte sich ein etwas zu hoher $\xi$-Wert errechnet. Außerdem enthalten die alten Daten von QUETELET (1871), die ich der damaligen Auswertung zugrunde legte, nicht den erst später erkannten Wachstumsschub während der Pubertät. Für die neue Berechnung dienten daher zunächst nur die Wachstumsdaten bis zum zehnten Lebensjahr. Hierbei stellte sich heraus, daß die erhaltenen Parameter die Meßdaten bis zur Pubertät wiedergeben, während darüber hinaus sich zunehmende Abweichungen ergeben.

Durch die Begrenzung auf den pueralen Cyclus ergab sich eine Herabsetzung des einzusetzenden $\xi$-Wertes, der dann aber - ebenso wie bei den Ratten -- auch für den postpueralen Cyclus eingesetzt werden kann. So ergibt sich jetzt die Möglichkeit, das gesamte menschliche Längenwachstum bis zu seinem Abschluß mathematisch relativ einfach wiederzugeben. Die grundsätzliche Möglichkeit, das menschliche Längenwachstum mit der neuen Funktion darzustellen, wird also durch die Korrektur nicht berührt, sondern ihr Anwendungsbereich nur erweitert.

Auch beim Menschen finden wir also die Unterteilung des postnatalen Wachstums in zwei Cyclen. Allerdings reicht beim Menschen die Gültigkeit embryonaler Parameter in das erste Lebensjahr hinein, und daher beginnt der puerale Cyclus erst gegen Ende des ersten Jahres, und es ist nicht möglich, mit den pueralen Parametern das Geburtsgewicht zu berechnen. In Tabelle 4 sind die erhaltenen Werte den Daten von Meredirh \& StUART (1962) für das Wachstum beider Geschlechter bis zur Pubertät und die errechneten Zahlen für das postpuerale Wachstum den Daten von Bowdich 

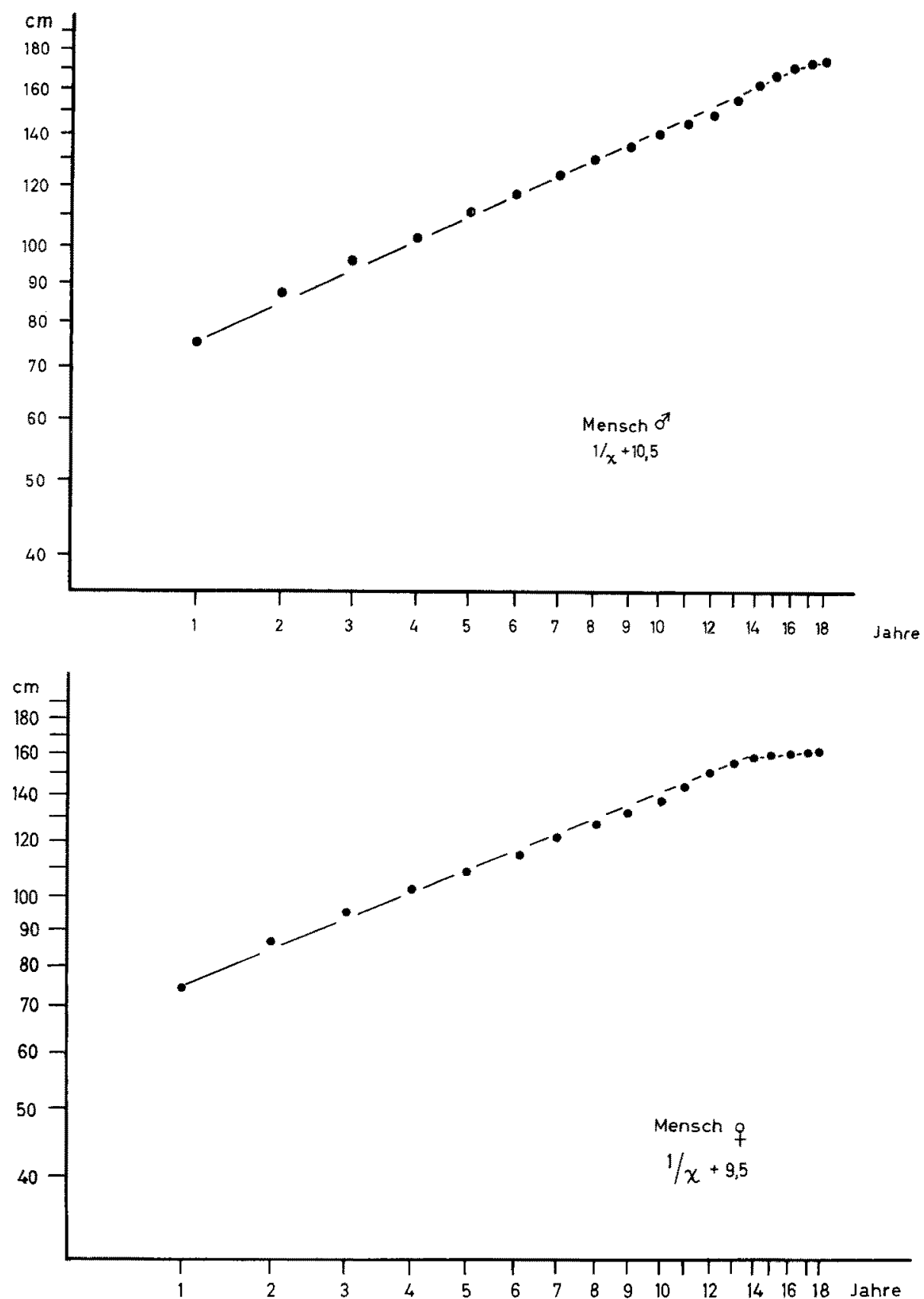

Abb. 3: Darstellung des Längenwachstums des Menschen gemäß der neuen Funktion. Auch beim Menschen tritt mit der Erreichung der Pubertät eine starke Verminderung der Wachstumsrate ein. Bei den nur bis zum 18. Lebensjahr reichenden Angaben von MEREDrTH \& STUART (1962) wird durch den frühen Eintritt der Reife beim weiblichen Geschlecht der postpuerale Cyclus deutlicher 
Tabelle 4

Längenwachstum des Menschen. Werte von MEredrth \& STUART (1962) (1 bis 16 Jahre) und BoDitch \& Baxter aus Backmann (1934) (16 bis 24 Jahre)

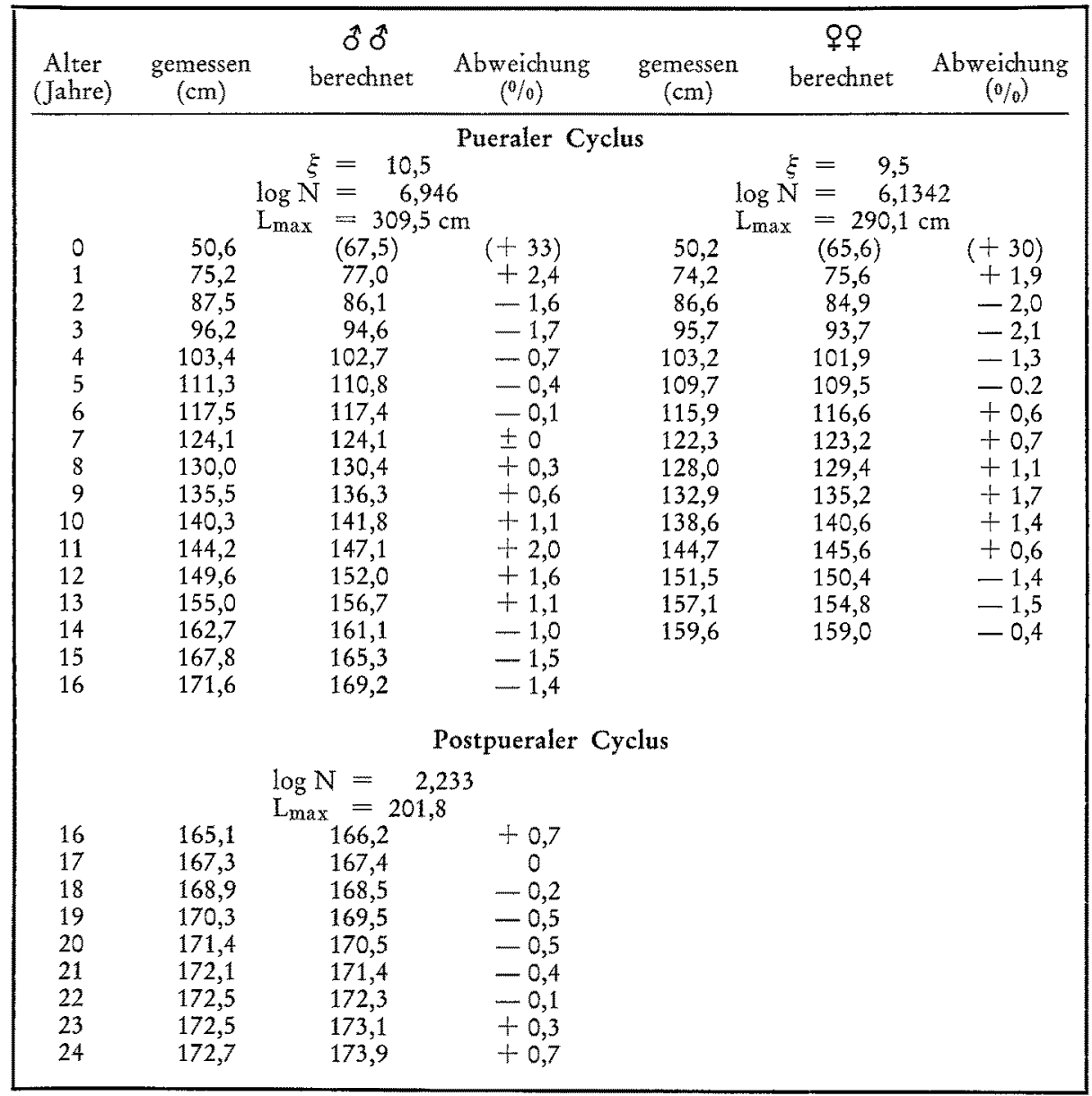

\& BAXTER gegenübergestellt. Es ergibt sich offensichtlich eine sehr gute Wiedergabe der menschlichen Wachstumskurye.

Auch das menschliche Embryonalwachstum läßt sich durch die neue Funktion gut wiedergeben, jedoch beträgt in diesem Falle der $\xi$-Wert nur etwa drei Wochen (Tab. 5). Die aus dem embryonalen Wachstum berechneten Werte stimmen über die Geburt hinaus noch bis zum dritten postnatalen Monat mit den Wachstumsdaten des Säuglings überein, erreichen aber auch dann noch nicht den Anschluß an das puerale Wachstum. Es läßt sich aber das anschließende Wachstum bis zum Ende des ersten Lebensjahres auf der Basis des embryonalen $\xi$-Wertes allerdings mit verminderter Geschwindigkeitskonstante wiedergeben; daher schlage ich für die Bezeichnung dieser Zeitspanne die Bezeichnung "postembryonaler" Cyclus vor; in Analogie zur Unterteilung der pueralen 
Tabelle 5

Längenwachstum des Menschen, embryonale Wachstumsperiode. Für das intrauterine Wachstum Werte von Marl, für das postnatale Wachstum Werte von Woodburg. (Aus BackMan 1934)

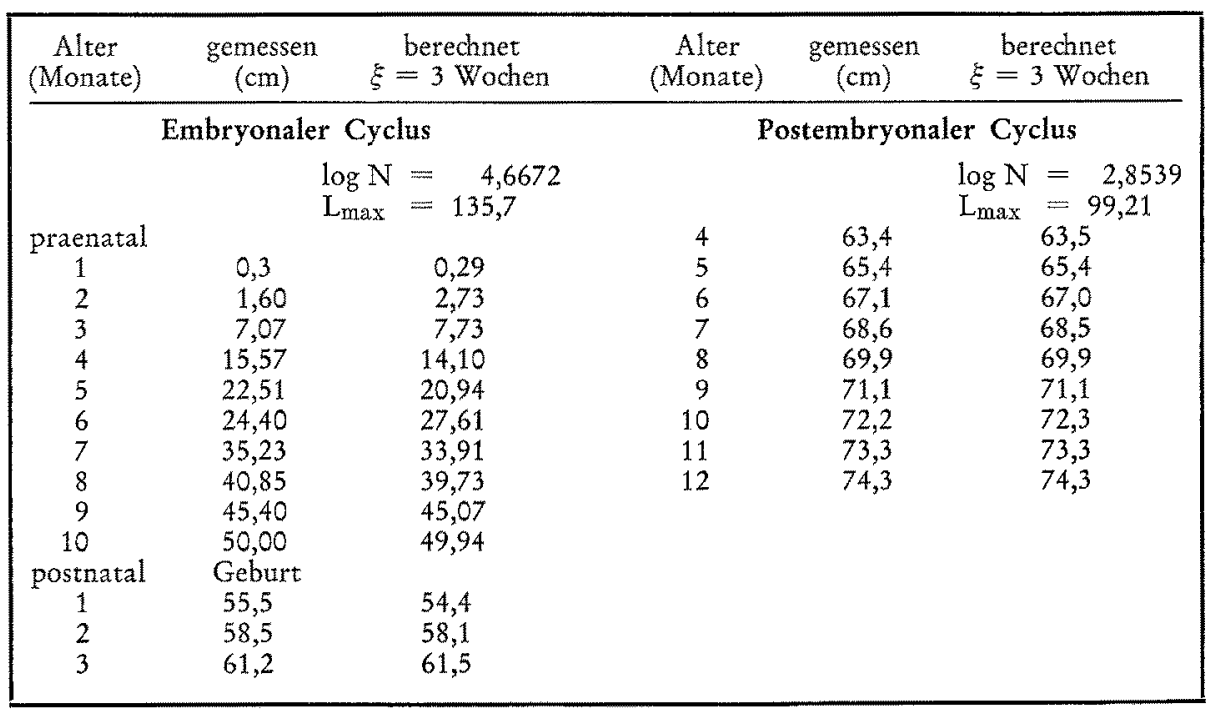

Wachstumsperiode. Erst mit Beginn des zweiten Lebensjahres erhalten die postnatalen Wachstumsparameter Gültigkeit. Die mathematische Auswertung auf Grund der neuen Funktion bestätigt also auch für das Wachstum die schon lange bekannte Tatsache, daß der Mensch noch in einem embryonalen Entwicklungszustand geboren wird.

Man erhält, wie wir sahen, mit der neuen Wachstumsfunktion über die ganze Wachstumsperiode - beginnend mit dem. Embryonalwachstum - einen sehr guten Anschluß an gegebene Daten. Für das postnatale Wachstum betragen die prozentualen Abweichungen maximal nur 2,4\%, die meisten Abweichungen liegen wesentlich niedriger. Die auffallend gute Ubereinstimmung zwischen gemessenen und berechneten Werten dürfte darauf beruhen, daß sich die Mittelwerte für das menschliche Wachstum auf ein sehr großes Zahlenmaterial stützen und daher ein hohes Maß an Zuverlässigkeit besitzen.

Eine genauere Betrachtung der Zahlen der Tabelle 4 oder der graphischen Darstellung ( $\mathrm{Abb}$. 3) zeigt nun aber, daß die $A b w e i c h u n g e n$ von den berechneten Daten nicht zufallsmäßig verteilt sind, sondern daß wir vielmehr Gruppen zu- und abnehmender Differenzen in ziemlich regelmäßiger Folge beobachten. Es lag daher nahe, die prozentualen Abweichungen der gefundenen von den berechneten Werten graphisch als Funktion des Alters darzustellen. Um die vorliegende Regelmäßigkeit deutlicher hervortreten zu lassen, wurden - bei dem gegebenen $\xi$-Wert - die beiden anderen Parameter so ausgerichtet, daß für das Alter 1 und 15 (beziehungsweise 13 im weiblichen Geschlecht) die Meßwerte resultieren. Die Abweichungen von den übrigen Punkten werden dadurch nicht wesentlich größer als bei der mathematisch optimalen Berechnung in Tabelle 4. Bei dieser Darstellung (Abb. 4) treten die regelmäßigen Schwankungen der Wachstumsgeschwindigkeit - im Vergleich zur Grundfunktion - deutlich 
hervor, und die eingezeichnete Sinusschwingung läßt erkennen, daß man sie in erster Näherung zum Beispiel durch eine Sinusfunktion darstellen könnte, die als Faktor der Grundfunktion zugefügt wird. Die das Wachstum des pueralen Cyclus überlagernde Schwingung stimmt in Amplitude und Periodenlänge bei beiden Geschlechtern überein
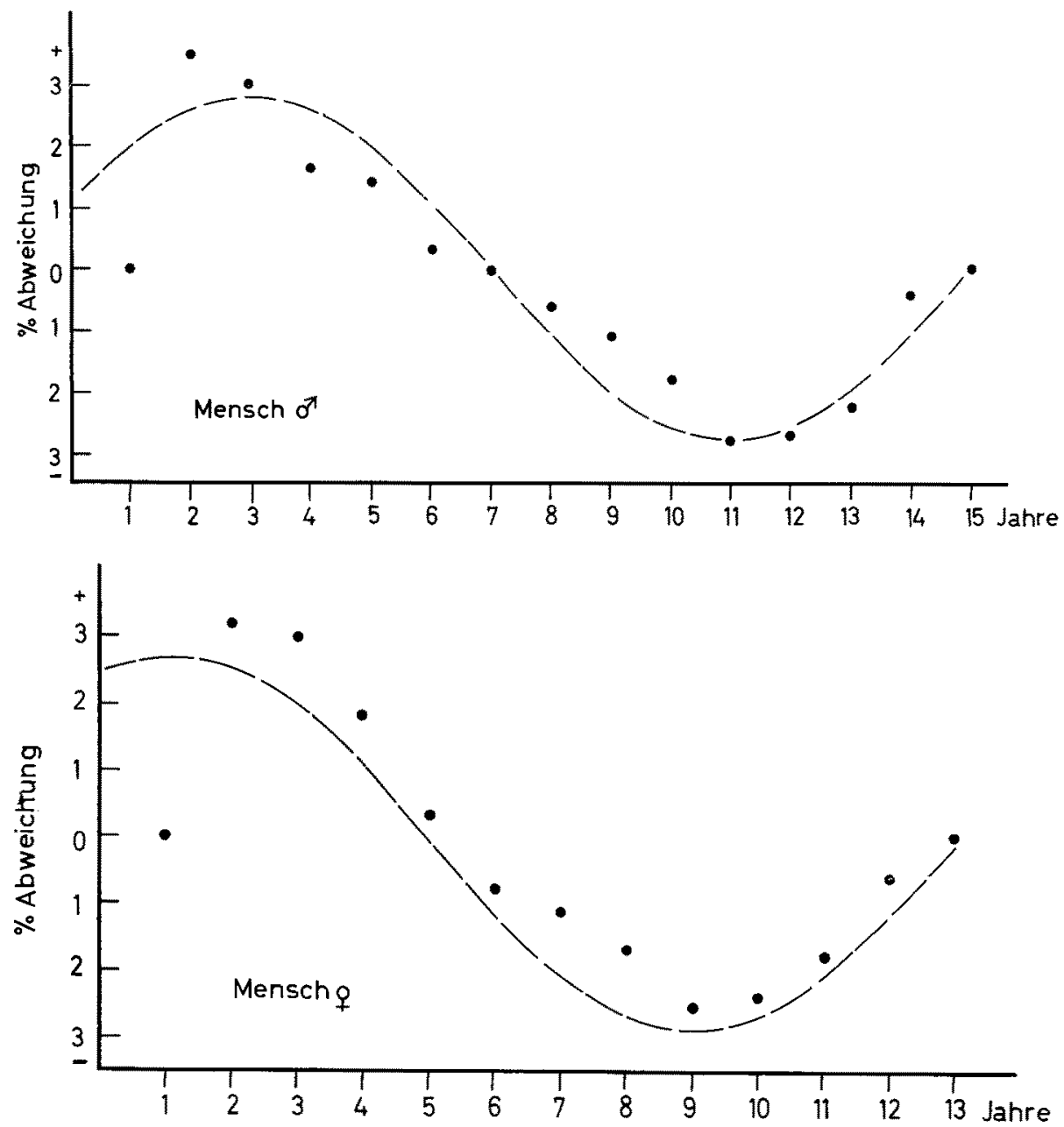

Abb. 4: Längenwachstum des Menschen. Graphische Darstellung der prozentualen Abweichungen der berechneten von den gemessenen Werten für den pueralen Cyclus. Zum Vergleich ist eine Sinusschwingung von ähnlicher Amplitude und Frequenz eingezeichnet

und unterscheidet sich nur hinsichtlich der Phasenlage zum Zeitpunkt der Geburt. Der Faktor wäre demnach: $\left(1+0,03 \cdot \sin 22,5^{\circ}(\chi+1)\right)$ im männlichen und $(1+0,03$. sin $22,5^{\circ}(x+3)$ ) im weiblichen Geschlecht. Der Winkel von $22,5^{\circ}$ gibt den auf ein Jahr entfallenden Anteil der Periodenlänge wieder und 0,03 die maximale prozentuale Amplitude. 
Tabelle 6

Berechnung des menschlichen Längenwachstums unter Berücksichtigung der Schwankungen der Wachstumsgeschwindigkeit; $\log \mathrm{N}$ und $\mathrm{L}_{\mathrm{max}}$ wurden so eingestellt, daß3 Anfangs- und Endwert mit den Meßwerten zusammenfallen

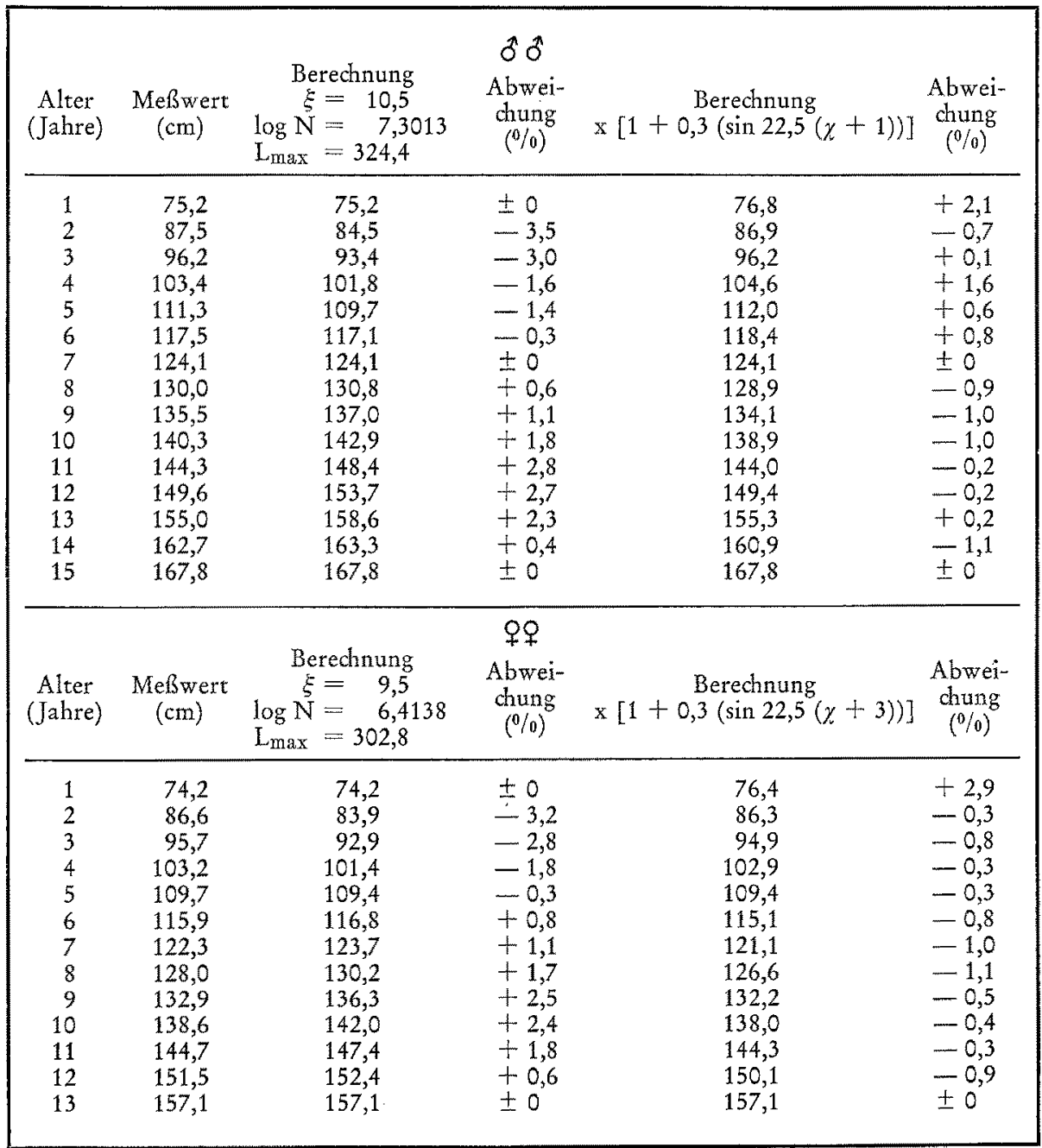

Durch einen solchen Zusatz wird naturgemäß die Zahl der bei der Wachstumsberechnung einzusetzenden Parameter verdoppelt, und man kann fragen, ob es nicht sinnvoller ist, in diesem speziellen Fall nach einer grundsätzlich anderen, einfacheren Lösung zu suchen. Abgesehen davon, daß im Augenblick eine solche Lösung nicht vorliegt, erscheint es mir sinnvoller, auch menschliche Wachstumsdaten zunächst in erster Näherung auf der Basis einer allgemein anwendbaren Funktion darzustellen, wie sie in der neuen Formel gegeben ist, und eine weitergehende Näherung durch geeignete $\mathrm{Zu}$ sätze zu erreichen. In welchem Umfang das möglich ist, zeigen die in Tabelle 6 auf 
Grund der groben Näherung berechneten Zahlen; nur die Altersstufe 1 zeigt noch bei beiden Geschlechtern eine erhebliche Differenz.

Die schon in der logarithmischen Wiedergabe erkennbare Unstetigkeit während des puberalen Wachstumsschubes dürfte es grundsätzlich unmöglich machen, das menschliche Wachstum durch irgendeine ganz einfache mathematische Formulierung darzustellen. Im übrigen sind die auftretenden systematischen Abweichungen auch keine Besonderheit des menschlichen Wachstums, sondern lassen sich in ähnlicher Weise auch beim Rattenwachstum und wahrscheinlich auch in andern Fällen nachweisen. Man kann also die neue Wachstumsfunktion nur als eine gute erste Näherung ansehen, die den prinzipiellen Verlauf tierischer Wachstumskurven wiedergibt.

In bezug auf das Längenwachstum des Menschen ist die übergelagerte Schwankung der Wachstumsrate dadurch von besonderem Interesse, daß sie uns eine Möglichkeit einer Deutung der hohen Wachstumsgeschwindigkeit vor dem Erreichen der Geschlechtsreife gibt. Wir finden zunächst eine sehr hohe Wachstumsrate zu Beginn des pueralen Cyclus bis zum ersten beziehungsweise dritten Lebensjahr. In der folgenden Zeit - bis zum neunten beziehungsweise elften Lebensjahr ist die Wachstumsgeschwindigkeit reduziert, und es folgt der Anstieg, der zum - rechnerischen - Normalwert führt. Durch die lange vorangegangene Periode reduzierten Wachstums wird die nun einsetzende Wachstumsbeschleunigung besonders augenfällig. Nach der vorgetragenen Deutung stellt der puberale Wachstumsschub also kein isoliertes Phänomen dar, wie es zum Beispiel DEMrNG (1957) annimmt, sondern ist nur ein Ausdruck für die während des ganzen pueralen Cyclus andauernde Schwankung der Wachstumsrate. Die gegebene Deutung scheint mir auch die Möglichkeit zu einer genaueren Analyse des Phänomens zu bieten.

Die gegebene Auswertung des menschlichen Wachstums bezieht sich - wie erwähnt - nur auf das Längenwachstum, das ganz allgemein einer mathematischen Beschreibung besser zugängig ist. Die Darstellung des menschlichen Gewichtswachstums führt - im Gegensatz zu den Verhältnissen bei der Ratte, wo es möglich war, das Gewichtswachstum darzustellen - zu unbefriedigenden Ergebnissen. Die Ursache hierfür liegt darin, daß das postnatale Wachstum des Menschen hinsichtlich der LängenGewichtsbeziehung nicht der allometrischen Funktion folgt, was bei der Ratte der Fall ist. Stellt man nämlich entsprechend der allometrischen Formulierung die Logarithmen von Länge und Gewicht in einem Koordinatensytem einander gegenüber, so ergibt sich für die postnatale Wachstumsperiode eine deutliche Kurve (Abb. 5). In auffälligem Gegensatz hierzu steht die geradlinige Anordnung der Punkte für die embryonale Wachstumsperiode, die auch bei dieser Art der Darstellung bis zum Ende des ersten postnatalen Lebensjahres reicht. Die allometrische Beziehung zwischen Länge und Gewicht gilt also für das embryonale Wachstum ungeachtet der starken Änderungen der Körperproportionen während dieser Zeit und den im Verhältnis zum postnatalen Wachstum starken Veränderungen in Länge und Gewicht. Es scheint mir hieraus hervorzugehen, daß die allometrische Längen-Gewichts-Beziehung keine rein geometrische Beziehung darstellt, sondern eine Wachstumsfunktion ist.

Die Längen-Gewichts-Beziehung für das postnatale Wachstum des Menschen wird also nicht durch die allometrische Funktion hinreichend beschrieben. Dagegen zeigte Roberts (1960), daß man diese Beziehung für den pueralen Cyclus, der ja den Haupt- 
teil des postnatalen Wachstums darstellt, bei semilogarithmischer Darstellung in guter Näherung durch eine Gerade darstellen kann. Hieraus wäre also die Funktion abzuleiten:

$$
\log w=a+p \cdot 1
$$

Der Logarithmus des Gewichtes nimmt beim Menschen also proportional dem linearen Wert der Länge zu. Hier scheint sich eine Sonderstellung des menschlichen Wachstums aufzuzeigen. Da die Länge in sehr guter Näherung mit der neuen Funktion für den

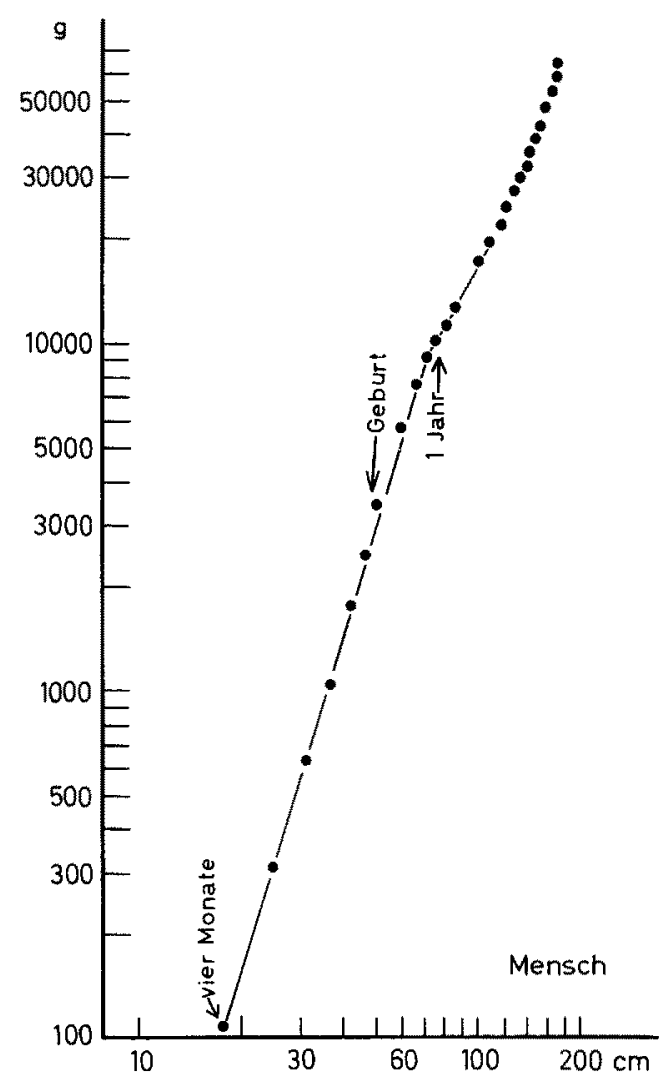

Abb. 5: Allometrische Darstellung der Längen-Gewichts-Beziehung des Menschen vom 4. Embryonalmonat bis zum 18. Lebensjahr. Auch die nicht angenommenen Daten für den 2. und 3. Embryonalmonat liegen in guter Näherung auf der Geraden. (Werte aus: WyLre, BlarNe \& AMIDON 1951 und Meredith \& STUART 1962)

pueralen Cyclus berechnet werden kann (Tab. 7), wäre mit Formel (5) auch das Gewichtswachstum zugängig. In ihr stellt p einen zahlenmäßigen Ausdruck für die Körperproportionen dar. a wäre das Gewicht bei der Länge Null, was mathematisch unsinnig ist. a ist also nur eine rein rechnerische Größe. Die Lösung ist mathematisch nicht befriedigend und auch zeitlich beschränkt. Die mathematische Darstellung des menschlichen Gewichtswachstums bleibt also ein noch zu lösendes Problem. 
Tabelle 7

Wiedergabe des Gewichts-Wachstums des Menschen als Funktion der Länge für den pueralen

Wachstums-Cyclus nach Gleichung (5) (Werte von Meredith \& STUART 1962)

\begin{tabular}{|rcccccc|}
\hline $\begin{array}{c}\text { Alter } \\
\text { (Jahre) }\end{array}$ & $\begin{array}{c}\text { Länge } \\
(\mathrm{cm})\end{array}$ & $\begin{array}{c}\text { Gewicht } \\
(\mathrm{kg})\end{array}$ & $\begin{array}{c}\text { Gewicht } \\
\text { berechnet } \\
=0,41972 \\
\mathrm{p}=0,77914\end{array}$ & $\begin{array}{c}\text { Länge } \\
(\mathrm{cm})\end{array}$ & $\begin{array}{c}\text { Gewicht } \\
(\mathrm{kg})\end{array}$ & $\begin{array}{c}\text { Gewicht } \\
\text { berechnet } \\
=0,39851 \\
\mathrm{p}=0,78762\end{array}$ \\
\hline 1 & 75,2 & 10,1 & 10,13 & 74,2 & 9,8 & 9,78 \\
2 & 87,5 & 12,6 & 12,63 & 86,6 & 12,3 & 12,28 \\
3 & 96,2 & 14,6 & 14,77 & 95,7 & 14,4 & 14,52 \\
4 & 103,4 & 16,5 & 16,80 & 103,2 & 16,4 & 16,66 \\
5 & 111,3 & 19,4 & 19,36 & 109,7 & 18,8 & 18,77 \\
6 & 117,5 & 21,9 & 21,64 & 115,9 & 21,1 & 21,04 \\
7 & 124,1 & 24,5 & 24,36 & 122,3 & 23,7 & 23,66 \\
8 & 130,0 & 27,3 & 27,08 & 128,0 & 26,4 & 26,27 \\
9 & 135,5 & 29,9 & 29,88 & 132,9 & 28,9 & 28,74 \\
10 & 140,3 & 32,6 & 32,57 & 138,6 & 31,9 & 31,92 \\
11 & 144,2 & 35,2 & 34,90 & 144,7 & 35,7 & 35,70 \\
12 & 149,6 & 38,3 & 38,49 & 151,5 & 39,7 & 40,45 \\
13 & 155,0 & 42,2 & 42,40 & 157,1 & 45,0 & 44,83 \\
14 & 162,7 & 48,8 & 48,68 & - & - & - \\
15 & 167,8 & 54,5 & 53,35 & - & - & - \\
\hline
\end{tabular}

Mit der quantitativen Erfassung von Wachstumsphänomenen sind wir aber schon in das Feld der mathematischen Beschreibung von Gestalten eingetreten. Grundsätzlich war das schon der Fall mit der Aufstellung der allometrischen Funktion, deren vielfältige erfolgreiche Anwendung uns zeigt, daß Größenbeziehungen im Organismus relativ einfachen mathematischen Beziehungen folgen und daß auch organische Gestalten einer mathematischen Behandlung zugängig sind.

\section{ZUSAMMENFASSUNG}

1. Zur Darstellung der Temperaturabhängigkeit biologischer Prozesse hatte ich eine neue Funktion vorgeschlagen, die formal der ArrhenIus-Funktion entspricht, jedoch eine höhere Bezugstemperatur als den absoluten Nullpunkt enthält. Es kann gezeigt werden, daß bei der Anwesenheit von Verzweigungen in den Reaktionsketten die Arrhenrus-Funktion ihre Gültigkeit verliert. Auf diese Tatsache könnte man die abweichende Bezugstemperatur der biologischen Temperaturfunktion zurückführen.

2. Die Temperaturabhängigkeit der Atmung von Salvelinus kann auch durch die von mir vorgeschlagene Wachstumsfunktion dargestellt werden. Hierbei ergibt sich eine wesentliche Vereinfachung der Darstellung.

3. Die Beziehung der neuen Wachstumsfunktion zur PüTrER-BerTalanfFy-Funktion wird diskutiert.

4. Am Beispiel des Wachstums der Albinoratte wird gezeigt, daß mit dem Erreichen der Geschlechtsreife eine Herabsetzung der Wachstumsgeschwindigkeit eintritt, ausgedrückt durch eine Herabsetzung des Werts für $\log N$. Hierdurch wird nach 
einiger Zeit das Wachstum rechnerisch praktisch unmeßbar gering. Es ergibt sich also die Notwendigkeit, das postnatale Wachstum der Ratte in einen vor der Geschlechtsreife liegenden "pueralen Cyclus" und einen sich anschließenden "postpueralen Cyclus" zu unterteilen. Der in die Berechnung einzusetzende $\xi$-Wert ist für beide Cyclen identisch.

5. Auch beim Längenwachstum des Menschen läßt sich diese Unterteilung des postnatalen Wachstums in einen pueralen und postpueralen Cyclus erkennen. Beiden liegt der gleiche $\xi$-Wert zugrunde. Die berechneten Werte zeigen aber eine systematische Abweichung von den Meßdaten, die sich näherungsweise durch eine Sinusfunktion wiedergeben lassen. Die hiermit zum Ausdruck kommenden Schwankungen der Wachstumsgeschwindigkeit erlauben eine Deutung des allbekannten puberalen Wachstumsschubes.

6. Im Gegensatz zum embryonalen Wachstum, das beim Menschen etwa bis zum Ende des ersten Lebensjahres reicht, folgt beim postnatalen Wachstum des Menschen die Längen-Gewichts-Beziehung nicht der allometrischen Funktion. In guter Näherung kann man - für den pueralen Cyclus - den Logarithmus des Gewichtes als Funktion des linearen Längenwertes darstellen.

\section{ZITIERTE LITERATUR}

BACKMAN, G., 1934. Das Wachstum der Körperlänge des Menschen. K. svenska Vetensk-Akad. Handl. 14, 1-145.

Beleyradek, J., 1930. Temperature coefficients in biology. Biol. Rev. 5, 30-58.

Bertalanffy, L. v., 1934. Untersuchungen über die Gesetzlichkeit des Wachstums. Arch. EntroMech. Org. 131, 613-652.

- 1938. A quantitative theory of organic growth. Hum. Biol. 10, 181-213.

- 1960. Principles and theory of growth. In: Fundamental aspects of normal and malignant growth. Ed. by W. W. Nowinski. Elsevier, Amsterdam, 137-259.

BrIDGES, C. H. \& Mullan, J. W., 1958. A compendium of the life history and ecology of the eastern brook trout. Fish. Bull. Mass. 23.

Brody, S. \& Proctor, R. C., 1932. Relation between metabolism and mature body weigth in different species of mammals and birds. Res. Bull. Mo. gr. Exp. Stn 166, 89-101.

Carlander, K. D. \& Ricker, W. E., 1962. Body length and weight in fishes. In: Growth, incl. Reproduction and morphological development. Comp. and ed. by P. L. Altman \& D. S. Dittmer. Fed. Am. Socs expl Biol., Wash., DC., 375-378 (Biol. Handb.).

CrozIer, W. J., 1924/25. On biological oxydations as a function of temperature. J. gen. Physiol. 7, 189-216.

Deming, J., 1957. Application of the Gompertz curve to the observed pattern of growth in length of 48 individual boys and girls the adolescent cycle of growth. Hum. Biol. 29, 83-122.

JANISCH, E., 1927. Das Exponentialgesetz als Grundlage einer vergleichenden Biologie. Abb. Theor. org. Entw. 2.

Job, S. V., 1955. The oxygen consumption of Salvelinus fontinalis. Univ. Toronto Stud. biol. Ser. (Publs Ont. Fish. Res. Lab. 73.) 61, 1-39.

JøRGENSEN, N. R., 1916. Undersøgelser over Frequensflader og Korrelation. Busck, Copenbagen, $214 \mathrm{pp}$.

KRÜGER, F., 1961. Uber den Exponenten der Temperaturfunktion biologischer Vorgänge und deren Größenabhängigkeit. Biol. Zbl. 80, 721-750.

- 1962. Uber die mathematische Darstellung des tierischen Wachstums. Naturwissenschaften 49,454 . 
- 1964. Uber die mathematische Form der biologischen Temperaturfunktion. In: TagBer. Gemeinsame Tag. d. Dt. Ges. f. Biophysik, Osterr. Ges. f. reine u. angew. Biophysik, Schweiz. Ges. f. Strahlenbiol., Wien 1964.

- 1964. Neuere mathematische Formulierungen der biologischen Temperaturfunktion und des Wachstums. Helgoländer wiss. Meeresunters. 9, 108-124.

- 1967. Zur mathematischen Darstellung des Rattenwachstums. Zool. Anz., Suppl. 30 (im Druck).

Meredith, H. V. \& STUART, H. C., 1962. Body measurements and organ weights: Man, North America. In: Growth, incl. Reproduction and morphological development. Comp. and ed. by P. L. Altman \& D. S. Dittmer. Fed. Am. Socs expl. Biol., Wash., DC, 334-336 (Biol. Handb.).

MurRay, H. A., 1925. Physiological ontogeny. 3. Weight and growth rate as function of age. J. gen. Pbysiol. 9,39 ff.

PütTer, A., 1911. Vergleichende Physiologie. Fischer, Jena, 721 pp.

- 1920. Studien über physiologische Ahnlichkeiten. 6. Wachstumsähnlichkeiten. Pflügers Arch. ges. Physiol. 180, 298-340.

Quetelet, L. A. J., 1871. Anthropométrie ou mesure des différantes facultés de l'homme. Bruxelles, Paris.

Rameaux, M. \& SarRus, P. F., 1837/39. [Application des sciences accessoires et principalement des mathématiques à la physiologie générale.] Bull. Acad. Méd., Paris 3, 1094-1100. (Zit. nach Thompson, D'Arcy W.: On grow th and form. 2nd ed. Vol. 1, 33.)

RoBERTs, D. F., 1960. Effects of race and climate on human growth as exemplified by studies on African children. Symp. Soc. Study buman Biol. 3, 58-72.

RuBNer, M., 1887. Die Gesetze des Energieverbrauches bei der Ennährung. Z. Biol. 19, $535-562$.

Schmalhatusen, I., 1925. Die individuelle Wachstumskurve von Paramaecium caudatum. Arch. EntMech. Org. 105, 711-717.

- \& BordzIlowsKaja, N., 1929. Über Analogie zwischen dem Wachstum der Organismen und Populationen. Arch. Entw Mech. Org. 115, 693-706.

Wyute, B., Blarne, F. \& Amidon, M. D., 1951. Correlation of weight, length and time factors in vital age. J. Obstet. Gynaec. Br. Commonw. 61, 193-196.

ZuCKER, L. M., 1962. Body measurements and organ weights: Rat. Pt 1. In: Growth, incl. Reproduction and morphological development. Comp. and ed. by P. L. Altman \& D. S. Dittmer. Fed. Am. Socs expl Biol., Wash., DC, 364 (Biol. Handb.).

- Hall, L., Young, M. \& Zucker, TH. F., 1941. Animal growth and nutrition, with special reference to the rat. Growth 5, 399-413.

\section{Diskussion im Anscbluß an den Vortrag KRÜGER}

WIESER: Wenn wir uns an die außerordentlich komplexen Modelle erinnern, mit denen hier während der Vorträge dieses Symposions Lebenserscheinungen abgebildet wurden, erhebt sich die Frage, welche Beziehung zwischen diesen und den 2-3-Parameter-Funktionen besteht, durch die Herr KRÜGER etwas so Komplexes wie das Wachstum von Organismen beschreiben will. Vermutlich ergibt sich als Antwort, daß einem biologischen System nicht die Wachstumsfunktion zugeordnet werden kann, sondern daß es viele Lösungen gibt. Jede Anderung der Um weltsituation mag zu Reaktionen des Systems führen, die es in eine andere Funktion transw portieren. Unstetigkeiten sind Wesensmerkmale biologischer Systeme und dürfen nicht einer pseudo-exakten Beschreibung geopfert werden.

KRÜGER: Ich erhebe nicht den Anspruch, endgültige Lösungen gegeben zu haben, aber es ist nicht eine Frage des Ermessens, ob sie geeignet sind oder nicht. Geklärt werden kann das Problem nur durch eine einwandfreie Auswertung exakter und ausreichender Meßdaten. Im biologischen Temperaturbereich beschreiben die bislang vorgeschlagenen Funktionen einen sehr ähnlichen Kurvenverlauf, der also zumindest sehr verbreitet auftritt wie beispielsweise die 
KroGH-Kurve. Es besteht hier keine Veranlassung, mit vershiedenen Funktionen zu arbeiten; es bleibr nur zu prüfen, welche Funktion am besten den verschiedenen Ansprüchen, die an eine biologische Funktion zu stellen sind, gerecht wird. Von der von Ihnen zitierten logistischen Funktion ist schon seit langem bekannt, daß sie als Wachstumsfunktion nicht in Betracht kommt, da sie einen symmetrisch gelegenen Wendepunkt aufweist, was für Wachstumskurven sicher nicht zutriff; sie kann höchstens zur Beschreibung kurzer Wachstumsspannen dienen. Bei der Unbestimmtheit biologischer Meßwerte spielt die Spanne, die eine Funktion umfaßt, eine ausschlaggebende Rolle für die Beurteilung der Anpassung an den Kurvenverlauf. Sie ist wesentlicher als die Wiedergabe spezieller Meßreihen. An eine Wachstumsfunktion sind mehr Forderungen zu stellen, als die Wiedergabe von Wachstumsdaten; dadurch verringert sich die Zahl der mathematischen Möglichkeiten. In bezug auf den Anschluß an die allometrische Funktion, und die Lage des Wendepunktes - bei hinreichender Wiedergabe von Wachstumsdaten - ist meine Formel allen bisherigen Vorschlägen eindeutig überlegen.

KNöTIG: Herr Wreser hat Recht: die Lebenssituationen variieren. Es ist aber möglid, durch Einführung neuer Parameter eine Anpassung an die veränderten Situationen zu erreichen.

Scharf: Ohne Zweifel ist Herrn Krüger zuzustimmen, wenn er die Meinung vertritt, es sei wünschenswert, funktionelle Zusammenhänge möglichst einfach darzustellen und die Gleichungen für die rechentechnische Behandlung bequem zu formulieren. Doch sollte nicht übersehen werden, daß ein Qualitätsmerkmal für den speziellen Fall der Wachstumsfunktionen ihre Extrapolierbarkeit in beiden Richtungen ist. Das adulte Tier ist sozusagen im Verhältnis zur Eizelle "unendlich" viel voluminöser. Man muß also fordern, daß die Funktion für $y(0) \approx 0$ wird. Mit steigendem Argument muB die Funktion dem Mittelwert entsprechend der Endgröße zustreben. Man muß nicht so weit gehen, wie der junge Mathematiker Rascer in seiner Habilitationsschrift (Rostock 1964), der die Ermittlung der Zeitkonstante $y$ für die Funktion $y=A_{0}+A_{1}$ eyt durch iterative Lösung der nicht linearen Gleichung fordert, die man durch partielle Differentiation nach $\gamma$ erhält. Aber immerhin habe ich gefunden, daß man mindest durch Regressionsrechnung in 2 Stufen Gleichungen vom Typus $y=A_{0}+A_{1} \mathrm{e}^{\mathrm{t}}+\mathrm{A}_{2} \mathrm{y}^{\mathrm{t}}$ bestimmen muß (Scharf, Morph. J6. 108, 283, 1966). Sollen auch Unstetigkeiten oder Oscillationen einbezogen werden, muß man imaginäre Glieder mitfihren (SCHArF, Verh. Anat. Ges., Jena 61, im Druck). Solche rechentechnisch aufwendigen, aber immer noch einfachen Funktionen erfüllen die empirischen Werte sehr gut und sind extrapolierbar. Ganz ohne Differentialgleichungen ist das Wachstumsproblem nicht mathematisierbar.

KRÜGER: Mein Vorgehen ist anderer Art; die von Ihnen geforderte Extrapolierbarkeit ist nicht gegeben. Mir kommt es aber weniger auf eine exakte Wiedergabe spezieller Fälle an, als darauf, eine Formulierung zu finden, die gestattet, auf einer allgemeinen Basis möglichst langfristig Wachstumsvorgänge darzustellen. Den Beweis, daß so etwas möglich sein muß, liefert die allometrische Funktion, deren allgemeine Anwendbarkeit in der Zwischenzeit durch zahllose Beispiele belegt ist. Sie bildete für mich den Ausgangspunkt. Auch die allometrische Funktion ist nicht nach beiden Seiten extrapolierbar und weist Unstetigkeiten auf; sie hat sich aber trotzdem als wichtiges Hilfsmittel erwiesen. Mein Vorschlag beinhaltet praktisch die allometrische Funktion, in die als dritter Parameter die Zeit eingeführt worden ist. Gerade die enge Beziehung zur allometrischen Funktion dürtte sich in Zukunt als sehr wichtig erweisen. Die allometrische Funktion ist aber nur mit einfachen Formulierungen in Beziehung zu setzen. Der einfache Aufbau einer mathematischen Formulierung stellt natürlich keine unabdingbaren Forderungen dar, erleichtert aber ihre Anwendung.

KIEfer: (1) Sie haben Ihre Temperaturfunktion, die drei Parameter enthält, mit 4 Meßpunkten geprüf. Eine Ubereinstimmung erscheint dann recht wahrscheinlich. (2) In Ihrer Funktion scheint kein Platz zu sein für die oft gefundenen Maxima in der Temperaturkurve. (3) Ausgehend von physikalisch-chemischen Ubberlegungen (ARRHENIUs) erscheint die Ableitung für ein lebendiges System ungeeignet, da nach dem Negentropie-Prinzip der Zusammenhang von Information und Energie beobachtet werden muß. (4) Mathematische Einfachheit erscheint nicht als das rechte Kriterium für eine geeignete mathematische Beschreibung biologischer Vorgänge. 
Scharf: Herm Kiffer stimme ich darin zu, daß bei 4 gegebenen Punkten kein Problem der Ausgleichsrechnung mehr vorliegt.

KRÜGER: (1) Die vorgeschlagene Temperaturfunktion wurde an einem andern Beispiel abgeleitet und nur auf die Meßwerte von Jos für Salvelinus angewandt. Es wurden nicht nur 4 Meßpunkte dargestellt, sondern jeweils 4 Punkte auf 6 Kurven, die einen sehr unterschiedlichen Verlauf haben und nun durch eine einheitliche Funktion überraschend gut dargestellt werden können. 3 Parameter sind das Minimum für eine Kurvenbeschreibung! Jos selbst hatte keinerlei mathematische Interpretation seiner Meßergebnisse unternommen. Grundsätzlich wäre dies mit den Formulierungen von JøRGENSEN oder BELEHRADEK gut möglich gewesen. Wie ich im vergangenen Jahr zeigte, weist mein Vorschlag mathematisch eine gewisse Uberlegenheit auf. Bei den kurzen Kurvenstücken, die bei der biologischen Temperaturfunktion zur Verfügung stehen, ist die eindeutige Entscheidung, welche am besten geeignet ist, schwierig. In diesem Fall müssen Kriterien auf anderen Ebenen mit herangezogen werden. Bei Salvelinus war es die offensidhtiche Beziehung der Temperaturparameter zu den Wachstumsparametern, welche die anderen Funktionen nicht so deutlich erkennen ließen. Gegenüber den in der Biologie oft falsch und kritiklos angewandten $Q_{10}$ - und $\mu$-Werten erscheint mein Vorschlag als bessere Lösung. (2) Die Darstellung eines temperaturabhängigen Maximums gestattet meine Funktion nicht. In der Formulierung von JANISCH ergibt sich ein Maximum durch die Ubberlagerung zweier gegensinniger Exponentialfunktionen. Auf diese Weise läßt sich mit jeder Funktion ein Maximum darstellen. Wegen der zumeist starken Streuung der Werte jenseits des Maximums mödhte ich auf die Darstellung dieses Kurventeils verzichten. (3) Die Ableitung der neuen Funktion erfolgte empirisch durch die graphische Analyse gegebener Versuchsdaten. Die formale Übereinstimmung mit der ArrHenius-Funktion ergab sich erst nachträglich. Deshalb fehlt auch die Gaskonstante, deren Anwesenheit auf speziellen physikalisch-chemischen Zusammenhängen beruht. (4) Mathematische Einfachheit ist selbstverständlich kein entscheidendes Kriterium, aber ein Vorzug, wenn andere unabdingbare Forderungen erfüllt sind, wie die richtige Lage des Wendepunktes und die definierte Beziehung zur allometrischen Funktion. Die etwas schwierige Handhabung der BERTALANFFY-Funktion, welche ohne $Z$ weifel sehr gut ist, hat ihrer breiteren Anwendung sicherlich im Wege gestanden.

HeINmers: Growth, especially in higher organisms, is essentially controlled by the endocrine system, and temperature represents only a partial parameter. Its role is highly limited in organisms which have temperature control mechanisms and consequently operate at constant temperatures.

KRỦGER: Selbstverständlich steht das tierische Wachstum unter hormonalen Einflüssen, aber auch deren Aktivität ist eine Funktion der Zeit. Die Darstellung des Wachstums als eine Funktion der Zeit und nicht - wie bisher üblich - der Masse erscheint mir besonders wesentlich. Für die Darstellung der Temperaturabhängigkeit des Stoffwechsels homoithermer Organisnen kommt meine Funktion naturgemäß nicht in Frage, solange die Regelmechanismen in Funktion sind.

Hess: Ihre Definition eines stationären Zustandes, insbesondere im Hinblick auf den sogenannten Anabolismus und Katabolismus ist meines Erachtens nur schwer zu durchschauen. $\mathrm{Da}$ in einem Vielkomponenten-System in e in e mompartiment Anabolismus, in einem anderen Kompartiment Katabolismus yorherrschen kann, kann eine solche Definition keine spezifische Bedeutung haben.

KRÜGER: Wir müssen natürlich mit einer gewissen Unschärfe bei allen biologischen Meßdaten rechnen. Im Fall von Salvelinus handelt es sich um Punkte auf den Regressionsgeraden, die aus einer weiten Temperaturspanne berechnet wurden. Sie dürften daher recht zuverlässig sein. In allen Fällen handelt es sich bei den zugrunde gelegten Zahlen um statistische Mittelwerte, die man wohl als Soll-Wert betrachten kann. In gegebenen Grenzen sind sie reproduzierbar. Die Formulierungen stellen eine Bilanz-Berechnung der Summe der zahllosen Primärprozesse dar, die wir im einzelnen nicht übersehen können. Mit der Gegebenheit der Fließgleichgewichte muissen wir im Organismus ebenso rechnen, wie mit einander parallel verlaufenden Auf- und Abbauprozessen, deren quantitative Beschreibung eine Aufgabe der Zukunft ist. 